OPEN ACCESS

Edited by:

Jack Tsao,

University of Tennessee Health

Science Center (UTHSC),

United States

Reviewed by:

Audrey Lafrenaye,

Virginia Commonwealth University,

United States

Zhihui Yang,

University of Florida, United States

${ }^{*}$ Correspondence:

Ciaran S. Hill

ciaran.hill@ucl.ac.uk

Specialty section:

This article was submitted to

Neurotrauma,

a section of the journal

Frontiers in Neurology

Received: 10 January 2020

Accepted: 17 April 2020

Published: 12 May 2020

Citation:

Hill CS, Sreedharan J, Loreto A Menon DK and Coleman MP (2020) Loss of highwire Protects Against the Deleterious Effects of Traumatic Brain Injury in Drosophila Melanogaster.

Front. Neurol. 11:401

doi: 10.3389/fneur.2020.00401

\section{Loss of highwire Protects Against the Deleterious Effects of Traumatic Brain Injury in Drosophila Melanogaster}

\author{
Ciaran S. Hill ${ }^{1,2 *}$, Jemeen Sreedharan ${ }^{2,3}$, Andrea Loreto ${ }^{1}$, David K. Menon ${ }^{4,5}$ and \\ Michael P. Coleman ${ }^{1,2}$
}

${ }^{1}$ John van Geest Centre for Brain Repair, University of Cambridge, Cambridge, United Kingdom, ${ }^{2}$ The Babraham Institute, Cambridge, United Kingdom, ${ }^{3}$ Institute of Psychiatry, King's College London, London, United Kingdom, ${ }^{4}$ Division of Anaesthesia, Department of Medicine, University of Cambridge, Cambridge, United Kingdom, ${ }^{5}$ Department of Clinical Neurosciences, Wolfson Brain Imaging Centre, University of Cambridge, Cambridge, United Kingdom

Traumatic brain injury is a major global cause of death and disability. Axonal injury is a major underlying mechanism of $\mathrm{TBI}$ and could represent a major therapeutic target. We provide evidence that targeting the axonal death pathway known as Wallerian degeneration improves outcome in a Drosophila Melanogaster model of high impact trauma. This cell-autonomous neurodegenerative pathway is initiated following axon injury, and in Drosophila, involves activity of the E3 ubiquitin ligase highwire. We demonstrate that a loss-of-function mutation in the highwire gene rescues deleterious effects of a traumatic injury, including -improved functional outcomes, lifespan, survival of dopaminergic neurons, and retention of synaptic proteins. This data suggests that highwire represents a potential therapeutic target in traumatic injury.

Keywords: wallerian degeneration, traumatic brain injury, neuroprotection, axons, highwire

\section{INTRODUCTION}

Traumatic brain injury (TBI) is defined as an alteration in brain function, or other evidence of brain pathology, caused by an external force (1). It is a major cause of death and disability, and each year in Europe alone $\sim 2.5$ million people will experience TBI, 1 million of which will be admitted to hospital, and 75,000 will die (2). Various secondary injury cascades follow the initial primary insult of TBI, promoting ongoing neuronal cell loss. The secondary injury response is multifaceted and comprises a number of processes including Wallerian degeneration (WD) (3).

WD is an active, cell-autonomous death pathway that leads to degeneration of the distal axonal segment following transection (4). In some neuronal subtypes WD following axonal injury may lead to a progressive dying back of the proximal axon segment and death of the neuronal cell body (5). A related process, Wallerian-like degeneration, occurs through the same molecular mechanisms as WD but does not require a complete axonal transection and instead is typically characterized by an impairment of axonal transport (4). WD and Wallerian-like degeneration are both thought to play a role in secondary brain injury following TBI although complete axotomy that occurs at the time of an injury (primary axotomy) is uncommon, and the degree to which sub-axotomy level injuries subsequently impair axonal transport and induce Wallerian-like degeneration remains incompletely characterized (6-10). 
Activation of the WD pathway is fundamentally linked to the nicotinamide adenine dinucleotide NAD synthetic pathway (Supplementary Figure 1A). Nicotinamide mononucleotide adenylyl transferase (NMNAT) is a key enzyme in the WD/Wallerian-like degeneration pathway, converting nicotinamide mononucleotide (NMN) to nicotinamide adenine dinucleotide (NAD). NMNAT also displays chaperone function during stress responses (11-15). Following transection, the delivery of the axonal isoform of NMNAT (NMNAT2) from the neuronal cell body to the distal axon is compromised. As NMNAT2 is degraded NMN accumulates and levels of NAD fall $(13,16-18)$. This results in activation of the toll-like receptor adaptor protein; sterile motif-containing and armadillo-motif containing protein (SARM1) resulting in axon fragmentation (Supplementary Figure 1B) (19-23). This mechanism of axon degeneration is conserved across species. In Drosophila Melanogaster, only one isoform of NMNAT is present, this is called dNMNAT (drosophila NMNAT) and serves the functions of mammalian NMNAT 1-3 isoforms, including delay in Wallerian degeneration. NMNAT proteins are evolutionarily conserved and dNMNAT can directly substitute for NMNAT isoforms in mammalian systems (24-28).

Evidence for a role of WD/ Wallerian-like degeneration in TBI has emerged from rodent cortical-contusion injury studies. The first WD gene to be explored in a model of TBI was the slow Wallerian degeneration gene $\left(\mathrm{Wld}^{s}\right)$. This gene encodes a mutant protein $\left(\mathrm{WLD}^{s}\right)$ that can substitute for NMNAT, and $\mathrm{Wld}^{\mathrm{s}}$ expressing mice demonstrate less motor and cognitive impairment following a cortical-contusion injury (29). Similarly, knockout of SARM1 was associated with reduced neuronal loss and cognitive impairment following CCI (30). These studies suggest that therapeutic modulation of the Wallerianlike degeneration pathway may be possible, and potentially could improve outcomes from TBI (3).

Another tractable context to explore the biology of WD is Drosophila Melanogaster. Loss-of-function mutations in the highwire (hiw) gene in D. Melanogaster are associated with a strong delay in axon degeneration following transection both in vitro and in vivo $(24,31-33)$. Delayed WD is also seen with mutations in the mammalian ortholog of hiw; PHR1 $(4,34,35)$. The hiw gene encodes a large 5233 amino acid protein with E3 ubiquitin ligase activity that modulates levels of dNMNAT $(24,31,35,36)$. Hiw also has presynaptic regulatory activity that is required to control excess synaptic growth at the neuromuscular junction $(31,36,37)$. To investigate for potential modifiers of brain trauma we utilized a model of high impact trauma (HIT) in D. Melanogaster in which WD pathways show extensive conservation with mammalian species $(4,19,20,35,38-40)$. Given the role of hiw in dNMNAT depletion and subsequent axon degeneration, and the assessment that Wallerian-like degeneration may contribute to the secondary brain injury seen in TBI, we hypothesized that flies with a null mutation in hiw $\left(h i w^{\Delta N}\right.$ ) would show protection against the effects of TBI. We show that $h i w^{\Delta N}$ flies showed relative protection against longterm mortality and cell death. Brain vacuolation and necrotic cell death occurred regardless of genotype-suggesting that the hiw mutants still underwent a neurodegenerative process but there was reduced presynaptic marker depletion and dopaminergic (PPL1) neuron loss. This suggests that a subset of vulnerablebut functionally important- neurons may be rescued by hiw loss-of-function. This translated to a preservation of normal behavioral measures. These findings suggest that hiw and its mammalian ortholog PHR1 are potential therapeutic targets in experimental and clinical TBI.

\section{MATERIALS AND METHODS}

\section{Drosophila Melanogaster Stocks and Conditions}

Hiw mutant hiw ${ }^{\Delta N}$ and control hiw ${ }^{W T}$ (FRT ${ }^{19 A}$ ) flies were obtained from Marc Freeman (University of Massachusetts). Newly enclosed flies were collected daily, separated by sex, into vials of 20-35 flies, and aged for experimental use. All experiments were conducted on flies aged 1-4 days unless otherwise stated. All flies were maintained at a constant $25^{\circ} \mathrm{C}$ temperature and humidity, in glass vials with standard agar/cornmeal/yeast feed. Flies were exposed to a $12 \mathrm{~h}$ light-dark cycle. Feed was changed in all vials once every 14 days or sooner as required. All experiments were conducted exclusively on male flies in order to avoid confounding effects relating to the female reproductive cycle.

\section{High Impact Trauma Device, Injury Calibration, Incapacitation Rates, and Intestinal Barrier Dysfunction}

Flies were subjected to a standardized impact with the HIT device. After injury, vials were laid on their side and flies were given a minimum of $10 \mathrm{~min}$ to recover motility before being transferred to a glass vial containing standardized feed. All polystyrene vials were discarded after a single use. The severity of injury was calibrated in hiw ${ }^{W T}$ flies by assessing the death rates $24 \mathrm{~h}$ following HIT when the angle of initial deflection, and thus recoil force, was adjusted. Incapacitation rates were recorded by assessing the percentage of flies that failed to show signs of purposeful movement within 20 seconds of initial impact. To evaluate intestinal barrier dysfunction following HIT flies were transferred to feed containing dissolved Brilliant Blue FCF dye (\#80717, Sigma). After $24 \mathrm{~h}$ the percentage of flies that had blue food dye dispersed outside of the abdominal cavity and proboscis were counted.

\section{Early Death Rate and Long-Term Survival Assay}

To assess for variation in early death rates we exposed flies to a HIT at a standardized time of day $(09: 00 \mathrm{~h})$. Any flies dying immediately or within the first $24 \mathrm{~h}$ of a HIT were considered to have died of the undifferentiated primary effects of a HIT. Dead flies were removed and all remaining live flies were transferred to new vials and long-term survival was monitored. A daily count of number of fly deaths was conducted in all vials for the lifetime of all flies. Dead flies were discarded every day. 


\section{Rapid Iterative Negative Geotaxis (RING) Assay and Flight Assay}

A custom made rapid iterative negative geotaxis (RING) device was manufactured and used to measure negative geotaxis/climbing ability as a behavioral measure of motor function $(41,42)$. Flies were gently transferred to fresh empty polystyrene vials without anesthesia with a maximum density of 25 flies per vial. Groups of up to 6 vials were inserted into the RING device, and after $5 \mathrm{~min}$ for the flies to adjust to the environmental change the device was tapped three times to settle flies to the bottom of the vials. Exactly $5 \mathrm{~s}$ after the last tap a photograph was taken to assess the height climbed. The head of the fly was the reference point for the climbing height achieved. Maximum height achieved was graded into $5 \mathrm{~mm}$ intervals, flies that climbed $<5 \mathrm{~mm}$ were scored zero, and any fly that exceeded $50 \mathrm{~mm}$ was awarded the maximum score was $5 \mathrm{~cm}$. The average height achieved for the vial was calculated. This was repeated 3 times at $60 \mathrm{~s}$ intervals and an average score given for that vial. The climbing ability was calculated on a vial by vial basis. For the flight assay, flies were anesthetized on ice for exactly 5 min then the flat of a 30G 1" needle (\#Z192368, Sigma) was attached to the anterior notum of a fly just posterior to the neck using clear nail varnish, leaving flight muscles unimpeded. Flies were given $15 \mathrm{~min}$ to fully recover. Needles were fixed in place under a video microscope. If required then a gentle mouth-blown puff of air was used to stimulate flight and the flying time was recorded for $30 \mathrm{~s}$ per fly for analysis. This was repeated 3 times per fly and the average of time spent in flight was calculated for each condition. This was a terminal assay. We maintained standardized conditions using the same experimental setup for each range of experimental condition in both the climbing and flying assay. The same experimenter conducted all experiments in a standardized fashion, in a single laboratory room, at the same time of day (09:00), and with the same equipment and environmental conditions-including room lighting and 25 degrees temperature).

\section{Haematoxylin and Eosin Histology, and Vacuole Counting}

Anesthetized flies were submerged in cold 1x PBS, the proboscis and rostral trachea were removed, and the amputated heads gently rocked in fresh ice cold $4 \%$ paraformaldehyde solution for $45 \mathrm{~min}$. Brain extraction is not necessary for high-quality H\&E analysis so brains were stained in situ within the cuticle. The tissue was alcohol dehydrated, xylene washed, and embedded in paraffin for serial sectioning with a microtome (Leica RM2235) at a thickness $7 \mu \mathrm{M}$. Sections were mounted on poly-L-lysine coated slides (P0245, Sigma). Wax was removed with a xylene bath then alcohol washes before haematoxylin and eosin staining, and application of coverslips. After blinding, three representative coronal sections were examined from a central brain region that included the medulla using brightfield microscopy. The average number of $\geq 5 \mu \mathrm{M}$ vacuoles per slice in each brain was calculated. Vacuoles were measured at the greatest diameter-microscopy photographs were analyzed using image J software (v1.51n). All image analysis was performed with the investigator blinded to experimental conditions.

\section{Immunohistochemistry}

Fly brains were dissected in cold $1 \mathrm{x}$ PBS and fixed in $4 \%$ paraformaldehyde-PBS for $30 \mathrm{~min}$. Brains were dissected for immunohistochemical analysis as penetration of antibody through the waxy cuticle is limited and would otherwise be inaccurate. Samples were washed in 1x PBS with $0.3 \%$ Triton X100 (\#T8787, Sigma) and blocked for $1 \mathrm{~h}$ at room temperature in 1x PBS with 0.3\% Trition X-100 and 1\% BSA (\#9647, Sigma). Brains were incubated in primary antibody diluted with blocking solution for $72 \mathrm{~h}$. After washing and incubating in a fluorescent secondary antibody solution for $4 \mathrm{~h}$, samples were washed and mounted between two coverslips in ProLong diamond antifade mountant (\#P36965, ThermoFisher). Confocal images were acquired on a Leica imaging system, at $z$-stack intervals not greater than $0.6 \mu \mathrm{M}$ and blinded for analysis. Primary antibodies used were mouse Tyrosine-Hydroxylase (anti-TH) antibody 1:100 (TH-antibody, \#22941, Immunostar Inc.) for the PPL1 cluster. Secondary antibodies were goat anti-mouse IgG (H+L) Alexa Fluor 488 (\#A11034, ThermoFisher), and donkey anti-rabbit IgG $(\mathrm{H}+\mathrm{L})$ Alexa Fluor 594 (\#\#21207). All image analysis was performed with the investigator blinded to experimental conditions. For each brain a Z-stack was taken that covered the entire extent of the fixed brain. Each Z-stack was set at a slice thickness of $0.5 \mu \mathrm{m}$. There were 1 or 2 regions of interest per brain depending on the quality of dissection. The PPL1 cluster is identified by its location and its characteristics major neuropil projections to the mushroom bodies and vicinity, this is clearly different from neurons in other adjacent clusters (43). A total of 10-12 PPL1 clusters were used per condition, this equates to 5-12 brains. All brains were immunolabelled using a standardized method in parallel at a single time point. Image settings were kept constant throughout all analysis. Images were analyzed using image J software (v1.51n).

\section{Immunoblotting}

Fifteen whole fly heads were collected and homogenized in Laemmli sample buffer and centrifuged 13,000 rpm for $5 \mathrm{~min}$. Supernatant protein lysates were resolved by SDS-PAGE on Mini-protean 4-15\% SDS resolving gel (\#4561086, Bio-Rad) and transferred to Immobilon-P PVDF membrane (\#IPVH00010, Merck). They were blocked in a $1 \%$ BSA solution (\#9647, Sigma), then probed with the following primary antibodies: Bruchpilot 1:5000 (nc82, \#2314866, DSHB), Discs-large 1:10,000 (4F3, \#528203, DSHB), Neuroglian 1:5000 (BP104, \#528402, DSHB), and $\beta$ tubulin 1:5000 (E7, \#2315513, DSHB). Bands were detected with goat anti-mouse, and goat anti-rabbit horseradish peroxidase-linked secondary antibodies (\#1706515 and \#1721011, Bio-Rad) and Supersignal West Dura extended duration chemiluminescence substrate (\#34075, ThermoFisher). Immunoblotting was done in triplicate. Density of bands was quantified using Image J software (v1.51n). Analysis method included cropping a standard size rectangular band around the area of the blot of interest was selected and then the generated histogram was cropped with a straight line at the base of the 
intensity peak-the resulting area under the curve was then converted by image J to a numerical value which represented blot density and was used for data analysis. Blot density was compared to the density of the $\beta$ tubulin loading control.

\section{Flow Cytometry}

Five fly brains were dissected in cold 1xPBS solution and transferred to dissection media containing $7.5 \mathrm{mls}$ of DMEM (high glucose, HEPES, phenol-red free, \#21063029, ThermoFisher), $2.5 \mathrm{mls}$ of 10x trypsin (\#15400054, ThermoFisher), and 1\% BSA (\#9647, Sigma). Brains were washed in trypsin free dissection media, gently triturated using a $200 \mu \mathrm{L}$ pipette 30 times, then filtered through a $70 \mu \mathrm{M}$ strainer. The resulting solution was mixed in a 1:1 ratio with Annexin V \& dead cell solution (Annexin V \& 7-AAD, \#MCH100105, Merck), incubated at room temperature for $20 \mathrm{~min}$, then processed on the Muse Cell Analyser (Merck) using inbuilt analysis software. Dilutions in trypsin free dissection media we made as required. For positive controls brains were incubated in $200 \mathrm{mM}$ of Actinomycin D (\#A1410, Sigma) at $37^{\circ} \mathrm{C}$ for $6 \mathrm{~h}$ before processing. The average cell $/ \mathrm{ml}$ concentration was $3.0 \times 10^{5}$ with a range of $2.1 \times 10^{5}$ to $9.8 \times 10^{5}$ between groups. The Muse $\mathrm{T}^{\mathrm{TM}}$ Cell Analyzer system can detect cells sized between 2 and $60 \mu \mathrm{m}$. The flow cytometry data was obtained from 5,000 events (gated cells) per sample. The percentages of cells shown in the figures were calculated from the mean fluorescence intensity in each of the four quadrants. Cell size was thresholded at a cell size index of 1.5 to exclude cellular debris. Gating was kept constant for all experiments-it was set on the two-parameter dot plot as displayed. This is auto-optimized by the Muse ${ }^{\mathrm{TM}}$ Annexin V \& Dead Cell Kit (EMD Millipore).

\section{Statistical Analysis}

Statistical analysis and graph fitting was performed using Graphpad Prism 7.02 (GraphPad Software Inc.). Statistical tests and biological/technical repeats are listed in respective figure legends-as a minimum all experiments were repeated in triplicate. Significance threshold was taken as a $p$ value $<0.05$.

\section{RESULTS}

\section{A Drosophila melanogaster High Impact Trauma Device Produces a Quantifiable Injury That Demonstrates Genotype-Dependent Variation in Early Survival}

To model TBI in D. Melanogaster we utilized the HIT device. This consists of a spring-loaded attachment that holds a polystyrene vial of flies and inflicts a rapid acceleration-deceleration impact injury [Supplementary Figure 2A-image from Katzenberger et al. (38)]. The severity of injury correlated with the angle of initial deflection of the device spring. An angle of $90^{\circ}$ produced an average death rate of $22 \%$ at $24 \mathrm{~h}$ and $30 \%$ at 7 days postinjury in wild type ( $h i w^{W T}$ ) flies (Supplementary Figure 2B). External injuries were never seen at angles of $90^{\circ}$ or less but were common $(82 \%)$ at an angle of $135^{\circ}$, therefore the $90^{\circ}$ was chosen to represent a single severe HIT in subsequent studies. Our choice of HIT severity was also based on the observed incapacitation rate-defined as the proportion of flies that demonstrated a lack of purposeful movement $20 \mathrm{~s}$ or more following trauma. The incapacitation rate was comparable in both hiw ${ }^{W T}$ and $h_{i w}{ }^{\Delta N}$, but when compared to a HIT at $90^{\circ}$, incapacitation rate at $70^{\circ}$ was greatly reduced $(\sim 100 \%$ vs. $\sim 50 \%)$ and was less consistent (Supplementary Figure 2C). Given this more consistent evidence of neural injury, and the lack of evidence of systemic injury at $90^{\circ}$, this HIT protocol was therefore chosen to represent a single severe traumatic HIT in subsequent studies. In order to minimize variability a single experimenter used the same HIT device with the same setup for all impact experiments. A visual marker was placed at the $90^{\circ}$ position (when viewed from a lateral viewpoint) this allowed an exact reproducible spring angle each time.

The $h i w^{\Delta N}$ flies have previously been reported as having synaptic overgrowth at neuromuscular junctions but are otherwise phenotypically normal $(31,36,37,44)$. We found that in uninjured flies the genotype ( $h i w^{W T}$ or $h i w^{\Delta N}$ ) did not have any influence on long-term survival, indicating no significant effect of the hiw null allele on baseline viability. HIT resulted in an incapacitation rate that was the same in both hiw ${ }^{W T}$ and $h i w^{\Delta N}$ and $24 \mathrm{~h}$ following a HIT both hiw $w^{W T}$ and $h i w^{\Delta N}$ flies demonstrated reduced survival compared to uninjured flies (Supplementary Figure 2D). However, notably the early death rate was greater in $h i w^{\Delta N}$ flies. The cause of this small excess burden of death was not determined by this study. This may reflect non-TBI related causes related to the constitutive loss of hiw expression. Increased mortality following HIT could also be due to traumatic intestinal barrier dysfunction. To exclude this possibility flies were exposed to colored food post HIT and examined for leakage of food dye (45). This demonstrated very low rates of intestinal barrier breakdown regardless of genetic background, supporting the conclusion that the HIT severity we applied did not cause gross thoraco-abdominal trauma (Supplementary Figure 2E).

\section{High Impact Trauma Induced Long-Term Mortality Is Reduced and Functional Impairment Rescued by Loss of highwire}

To determine if loss of hiw could protect against long-term effects of TBI we examined survival in animals that lived beyond the initial $24 \mathrm{~h}$ post injury period. Injury caused a significant reduction in long-term survival in both hiw ${ }^{W T}$ and $h_{i w}{ }^{\Delta N}$ animals compared to uninjured controls. However, hiw ${ }^{\Delta N}$ animals demonstrated significantly increased survival following injury compared to hiw ${ }^{W T}$, particularly from $\sim 20$ days post injury (Figure 1A). To determine if loss of hiw could rescue injury-induced climbing deficits we used the rapid iterative negative geotaxis (RING) assay (Supplementary Figure 2F) (41, 42). This demonstrated a significantly reduced climbing ability after HIT in hiw ${ }^{W T}$ flies, which was attenuated in those with a $h i w^{\Delta N}$ deletion (Figure 1B). We also examined motor function by measuring flying ability. Flight behavior has been shown to vary with the level of protocerebral anterior medial dopaminergic 

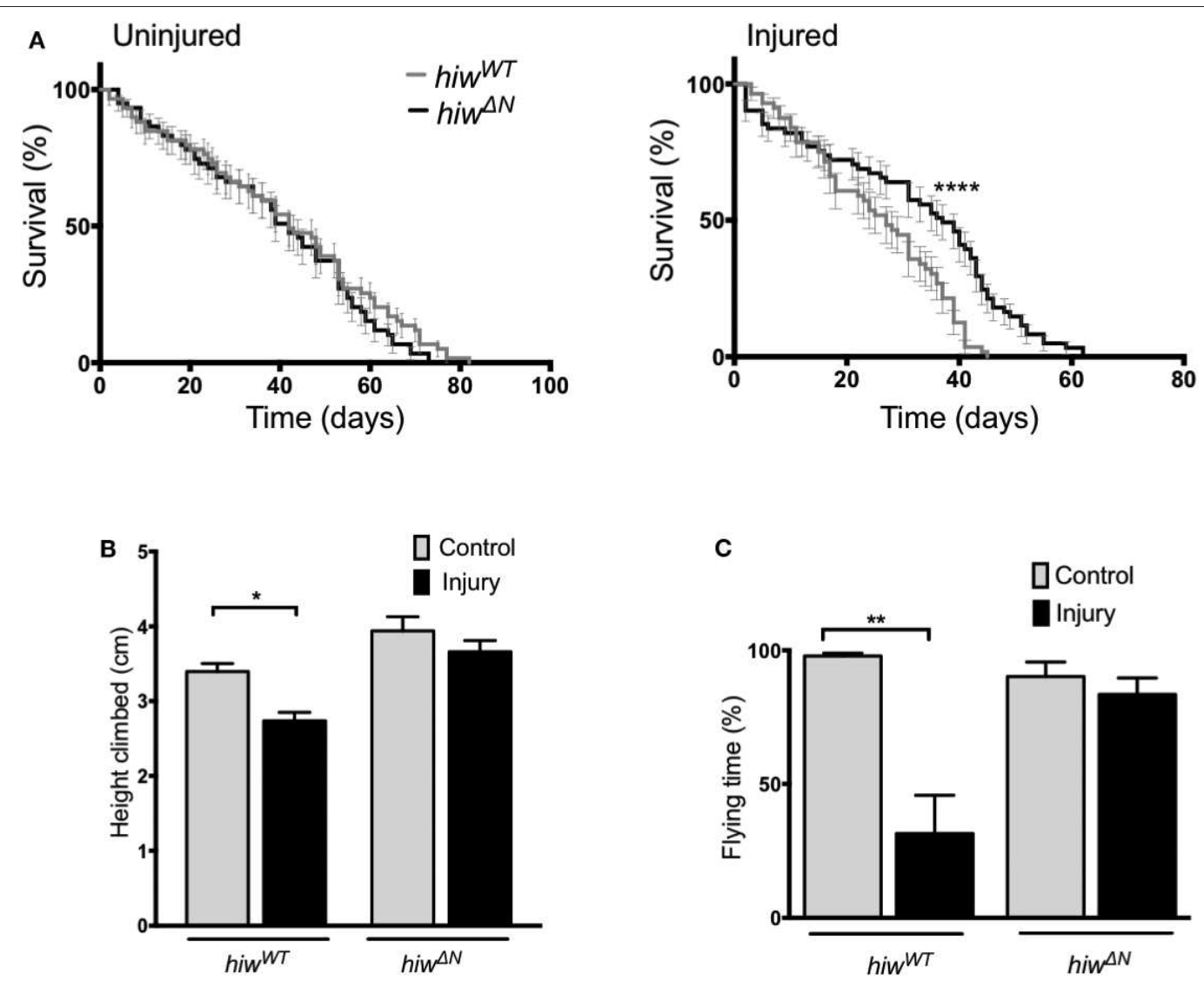

FIGURE 1 | Long-term mortality, climbing, and flying ability are relatively preserved in highwire mutants. (A) Mortality rates over lifetime of flies in hiw ${ }^{\Delta N}$ and hiwWT flies. $n=6$ vials of 20-35 flies per condition. Statistical analysis was with Log-rank test. ${ }^{\star \star * \star} p \leq 0.0001$. (B) Reduction in climbing ability compared to baseline in injured versus uninjured hiw ${ }^{\Delta N}$ and hiw ${ }^{W T}$ flies at 45 days post injury. $n=6$ vials of 35 flies per condition. Statistical analysis was with two-way ANOVA test. ${ }^{*} p \leq 0.05$. (C) Percentage of time spent in flight over a 30 s period in injured versus uninjured hiw ${ }^{\Delta N}$ and hiw ${ }^{W T}$ flies at 7 days post injury. $n=10-12$ per condition. Statistical analysis was with two-way ANOVA test. ${ }^{\star \star} p \leq 0.01$. Error bars show standard error of the mean for all experiments.

neurons (46). At 7 days following injury hiw ${ }^{W T}$ show a significant reduction in the percentage of time they spend in flight over a $30 \mathrm{~s}$ period when compared to controls of their own genotype. This deterioration in flying activity after injury is strikingly diminished in the hiw ${ }^{\Delta N}$ flies (Figure 1C). Injured flies that do not have flight activity still have healthy looking wings, make frequent spontaneous wing movements, and engage in wing grooming behavior, suggesting that the failure to fly even when provoked by a stimulus of air is not simply as a result of a peripheral wing injury. This is supported by the cases where short periods of flight are initiated but the flies seem unable to maintain for a prolonged duration.

\section{Loss of highwire Reduces Neuronal Apoptosis}

In order to explore the mechanism of neuronal cell loss following HIT we performed flow cytometry of dissociated fly brains (Figures 2A,B). This demonstrated low-levels of baseline early apoptosis (Annexin $\mathrm{V}$ positive, $7 \mathrm{AAD}$ negative) and late apoptosis/cell death (Annexin $\mathrm{V}$ positive and 7 AAD positive) in hiw ${ }^{W T}$ and $h i w^{\Delta N}$ uninjured controls $(<0.5 \%)$. In contrast,
7 days following injury there was a rise in both early apoptosis, and late apoptosis/cell death by flow cytometry. This is direct evidence for brain injury following impact with the HIT device. The levels of early apoptosis were significantly lower in the hiw ${ }^{\Delta N}$ flies compared to hiw ${ }^{W T}$ following injury at 7 days, however the absolute difference was small $(\sim 0.5 \%)$. There were no significant differences in late apoptosis/death at either time point. This suggests that HIT causes a neuronal apoptotic cell death that is rescued by $h i w^{\Delta N}$ mutation.

\section{Dopaminergic Neuron Loss Is Attenuated by Loss of highwire}

To explore the mechanisms underlying the protective effects of hiw following TBI we performed histological studies on fly brains. We first looked for evidence of injury-induced neurodegeneration by quantifying brain vacuolation (38, 4749). Both hiw ${ }^{W T}$ and $h i w^{\Delta N}$ flies demonstrated an increase in vacuolation at 28 days following injury, though no attenuation of vacuolation was observed in $h i w^{\Delta N}$ flies (Figure 3A). We next examined a well-characterized subpopulation of dopaminergic neurons (protocerebral posterior lateral 1 cluster, 


\section{A Early apoptosis}

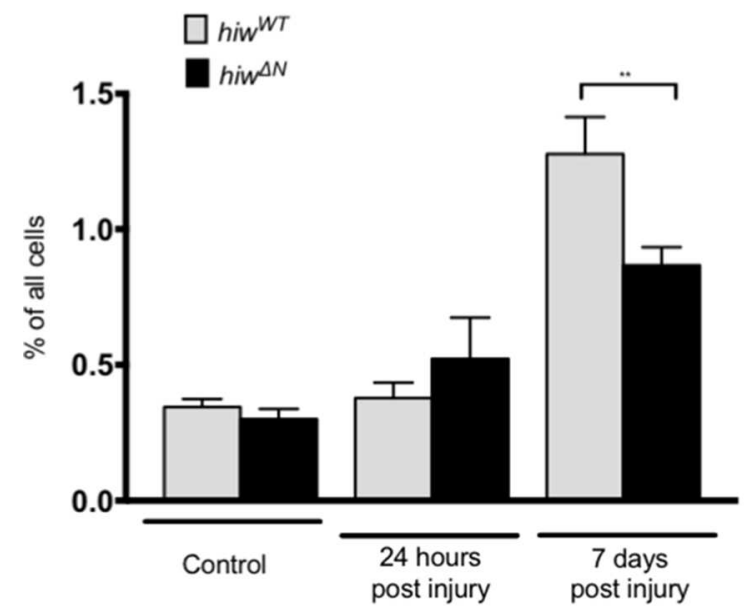

\section{Late apoptosis/death}

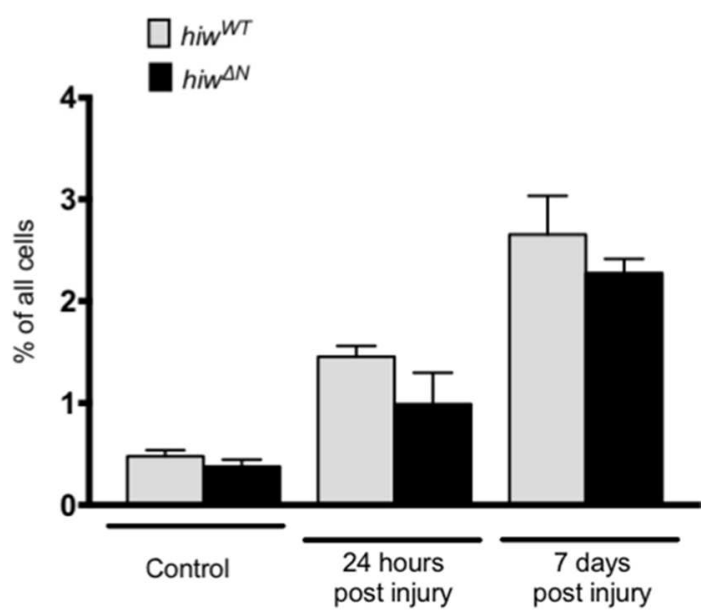

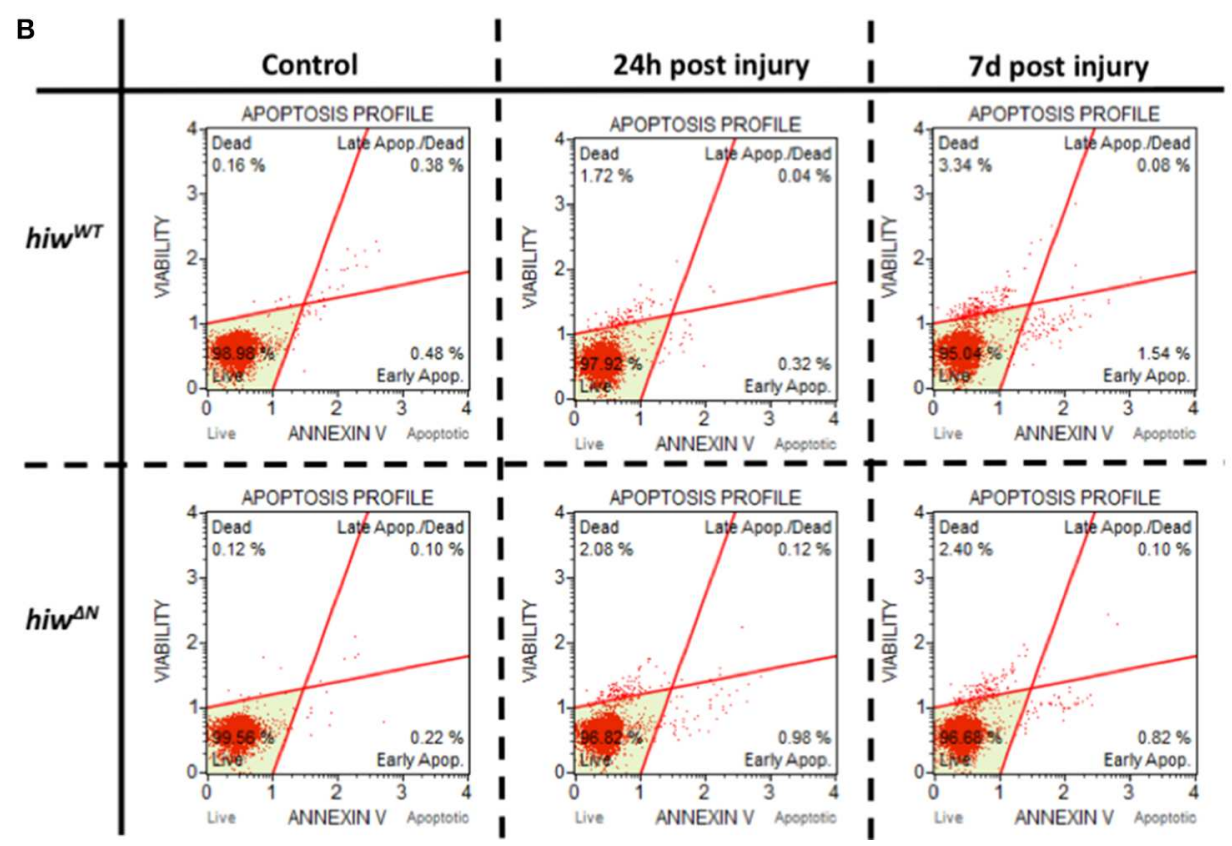

FIGURE 2 | Flow cytometry of dissociated drosophila brains shows necrosis but low levels of apoptosis after injury. (A) Flow cytometry demonstrating the percentage of cells undergoing early apoptosis, and late apoptosis/cell death following injury at $24 \mathrm{~h}$ and 7 days in hiw ${ }^{\Delta \mathrm{N}}$ and hiw ${ }^{\mathrm{WT}}$ flies. $n=9$. Error bars show standard error of the mean. Statistical analysis was with two-way ANOVA test. ${ }^{* \star} p \leq 0.01$. (B) Graphical representative flow cytometry data.

PPL1), which are involved in climbing and flying behavior (Figure 3B) $(46,50,51)$.

The number of neurons in the PPL1 cluster is remarkably consistent in wild-type flies, with an average of 12 neurons. Immunostaining for tyrosine hydroxylase $(\mathrm{TH})$ demonstrated a significant decrease in the number of neurons in the PPL1 cluster following injury in hiw ${ }^{W T}$ flies. However, no reduction in PPL1 neuron numbers was seen following injury in $h i w^{\Delta N}$ flies (Figures 3C,D).

\section{Loss of highwire Reduces Synaptic Protein Loss Following Injury}

In order to probe the nature of underlying cellular responses to the HIT we conducted western blots analysis using the pan-neuronal marker Neuroglian (Ngl), the presynaptic marker Bruchpilot (Brp), and the post synaptic maker Disc Large (Dlg) at $24 \mathrm{~h}$ and 7 days following injury. After injury there was a significant reduction in $\mathrm{Ngl}$ at $24 \mathrm{~h}$ in $h_{i w}{ }^{W T}$ indicating a generalized loss of neurons. This trend was also seen at 7 days 
A

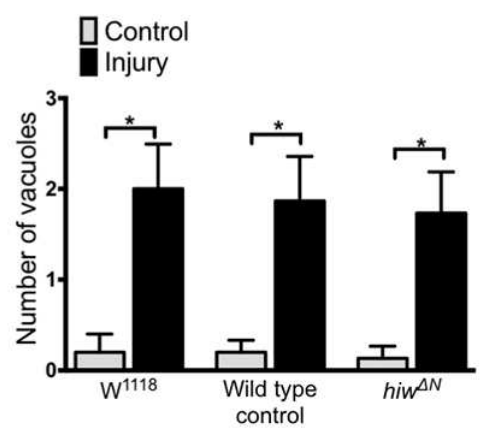

B

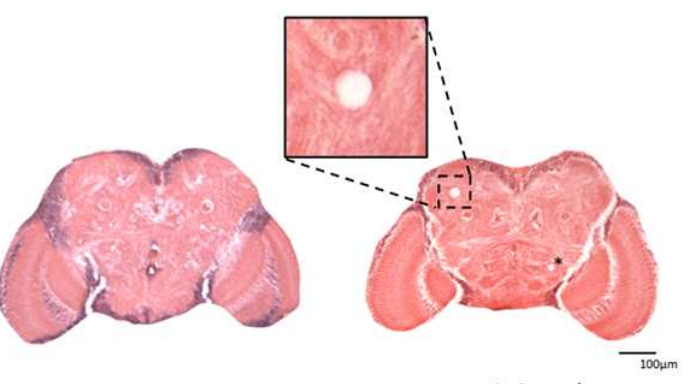

Uninjured

Injured

C
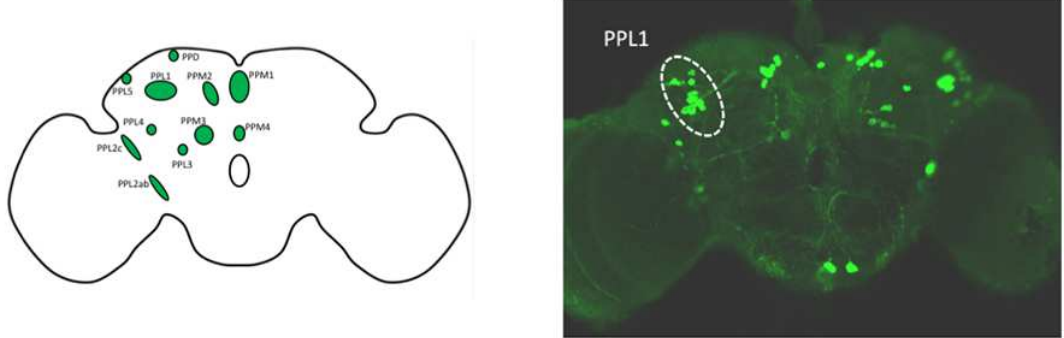

D

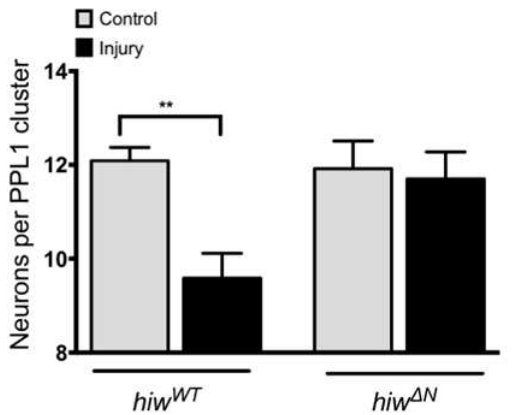

E

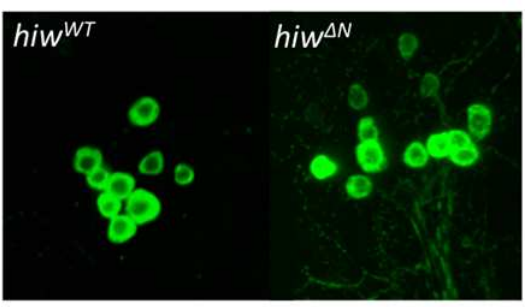

FIGURE 3 | Injured flies develop brain vacuoles regardless of genotype, but depletion of PPL1 cluster dopaminergic neurons that is prevented by highwire mutation. (A) Rates of vacuolation seen by hematoxylin and eosin staining in central brain regions at 28 in hiw ${ }^{\Delta \mathrm{N}}$ and hiw ${ }^{\mathrm{WT}}$ flies 28 days following injury. $n=5$ per condition. Error bars show standard error of the mean. Statistical analysis was with two-way ANOVA test. ${ }^{*} p \leq 0.05$. (B) Representative hemotoxylin and eosin stained brain sections from uninjured and injured hiw ${ }^{W T}$ flies. The insert shows a close up of a typical vacuole. A further small vacuole is marked by an asterix. (C) Schematic image 
FIGURE 3 | and TH stained whole brain mount showing the location of various dopaminergic neuronal groups including the PPL1 cluster. (D) Number of TH-positive PPL1 neurons in hiw ${ }^{\Delta N}$ and hiw ${ }^{W T}$ flies at 28 days post injury. $n=10-12$ clusters per condition. Error bars show standard error of the mean. Statistical analysis was with two-way ANOVA test. ${ }^{* *} p \leq 0.01$. (E) Representative PPL1 dopaminergic neuronal clusters showing depleted neuron numbers in injured hiw ${ }^{W T}$ flies (9 neurons) and preserved numbers in injured hiw ${ }^{\Delta N}$ flies (12 neurons).

but was no longer significant. In contrast, $h i w^{\Delta N}$ flies did not have a reduction in Ngl. Mirroring the Ngl loss, Brp was also reduced following injury at $24 \mathrm{~h}$, but this reduction persisted at 7 days with some progressive loss. As with ngl, the hiw ${ }^{\Delta N}$ flies were protected against Brp loss regardless of timepoint- and a nonsignificant trend suggesting a possibly increase was seen. Finally, Dlg levels were examined. Again, trends mirrored those of $\mathrm{Ngl}$ and Brp, with small falls seen in the hiw ${ }^{W T}$, and small rises in the hiw ${ }^{\Delta N}$ flies, but notably these were not significant in any case (Figure 4).

\section{DISCUSSION}

Our results provide support for the hypothesis that a mutation in the hiw gene $\left(h i w^{\Delta N}\right)$ demonstrates protection against the deleterious effects of HIT. The hiw ${ }^{\Delta N}$ mutation results in a complete loss-of-function of highwire, leading to delayed degradation of dNMNAT, a core step in the WD process. The hiw ${ }^{\Delta N}$ mutation has previously been shown to strongly delay WD both in vitro and in vivo $(24,31-33)$. In $D$. Melanogaster, the hiw ${ }^{\Delta N}$ mutation protected against several deleterious effects of a high-velocity impact trauma model. Deaths were significantly reduced in keeping with longerterm protection against secondary brain injury processes. Flies with $h i w^{\Delta N}$ mutation suffer less neurodegeneration following HIT as manifest by reduced PPL1 dopaminergic neuron loss. Dopaminergic neurons have previously been shown to demonstrate selective vulnerability following brain injury and in various neurodegenerative conditions including Parkinson's disease (50-53). Our investigation of dopaminergic neurons was limited to the PPL1 subpopulation. Although the TH stain marks other dopaminergic populations the PPL1 population is the best characterized and has the small variability in its neuronal number. This is not the case for other neuronal populationswhich are less consistent in their number, not as easy to reliably count due to variability in density, and not as well characterized in terms of relationship to function. The focus on PPL1 allowed us the confidence that our staining technique was reliable and comparable to pre-existing studies, and allowed us to robustly detect differences due to injury or genetic modification. However, as a result of this specific focus we cannot make comment about the effect on other neuronal populations. In order to try and address the limitation of only quantifying a small subpopulation we looked for more general evidence of neuronal loss and rescue with the vacuolation assay. Injury resulted in increased vacuolation in all genotypes but interestingly this was not rescued in the hiw ${ }^{\Delta N}$ mutants.

Given the well-characterized function of highwire in dNMNAT degradation, the mechanisms for the protection seen with hiw $^{\Delta N}$ mutation are likely to directly involve the Wallerian-like degeneration pathway, specifically through maintenance of dNMNAT levels in the axonal compartment and/or the cell body and delayed neuronal degeneration. As there is only a single dNMNAT isoform in D. Melanogaster, a decline in levels may cause death of both the axon and the cell body. An important future experiment would be to assess dNMNAT levels in a neuronally expressed HA-nmnat tagged fly system.

An alternative explanation would be that loss of highwire function is maintaining dNMNAT levels that are then functioning as a molecular chaperone, possibly through alleviation of prototoxic stress $(11,12,14,15,54)$. Given the nature of the model system we cannot completely exclude that the rescue of lifespan and behavior are not due to effects outside of the CNS, however, the lack of external injuries, the failure of the model to cause intestinal barrier dysfunction, and the initial period of recovery and normal behavior following injury argue against this. The $\operatorname{hiw}^{\Delta N}$ mutation is constitutive, therefore there may be unrecognized systemic effects beyond those we have characterized in the brain. This could be addressed in future studies by examination of a conditional mutant. We know that hiw ${ }^{\Delta N}$ flies have previously been reported as having synaptic overgrowth at neuromuscular junctions but are otherwise phenotypically normal $(31,36,37,44)$. We cannot completely exclude the possibility that there is a hitherto unrecognized underlying phenotypic difference that we, and previous researchers have failed to identify. As long as this possibility remains we would urge caution in interpreting the results of this study in isolation.

We did not find any alteration in the levels of synaptic proteins in the uninjured $h i w^{\Delta N}$ brains compared to wild-types in our analysis. This suggests that the synaptic overgrowth effects seen previously at the neuromuscular junction of $h i w^{\Delta N}$ do not occur in the brain. We demonstrated that HIT trauma resulted in an excess of apoptotic cell death that was partially rescued by $h i w^{\Delta N}$. This is an intriguing finding given that WD is a nonapoptotic mechanism of axon degeneration. One possibility is that absence of retrograde delivery of trophic factors by axonal transport could trigger apoptosis of cell bodies-this hypothesis requires further testing. Behavioral measures are less commonly used and well-defined in flies compared to mammalian systems. We chose to use two of the commonest and best characterized in climbing and flying assays. The ability of hiw ${ }^{\Delta N}$ to prevent injury induced climbing and flying defects may be explained by dopaminergic neuron rescue, and PPL1 neuronal loss has previously linked to climbing deficits $(46,51)$. The preferential presynaptic protein loss seen on Western blot analysis is a pattern that is established in neurodegenerative models (55, 56). dNMNAT has also been shown to maintain presynaptic 


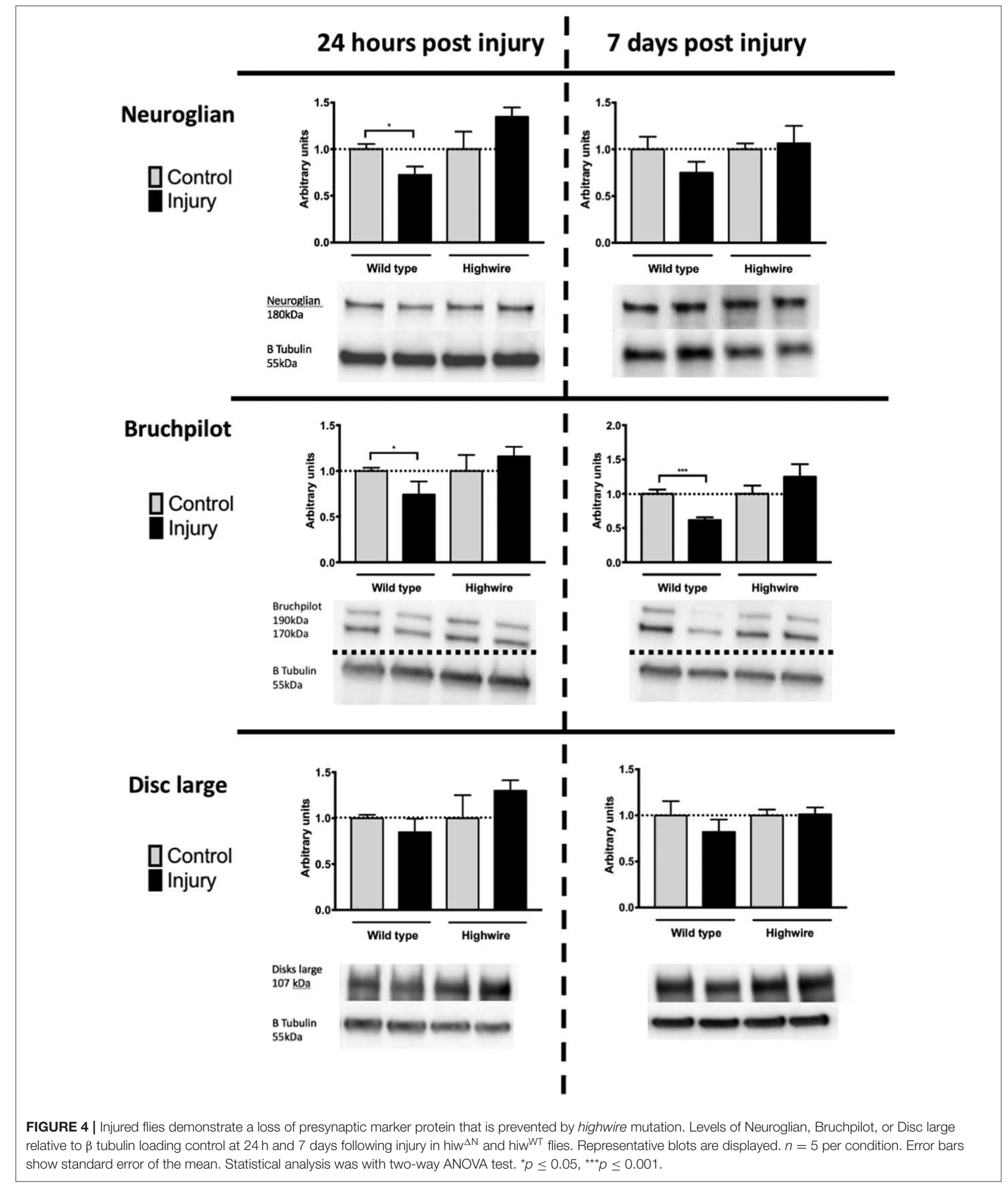

active zones by directly interacting with bruchpilot (57). This is consistent with our finding that bruchpilot was relatively preserved after injury in $h i w^{\Delta N}$. Together, the finding suggest that HIT causes apoptotic and selective neuronal cell death and presynaptic protein loss, and that highwire loss of function can partially rescue these effects and their functional consequences. 
This helps establish highwire as a potential therapeutic target in brain trauma.

\section{DATA AVAILABILITY STATEMENT}

All datasets generated for this study are included in the article/supplementary material.

\section{ETHICS STATEMENT}

The experimental protocol was approved by the Babraham Institute ethical review board, and all animal work was performed in accordance with the 1986 Animals (Scientific Procedures) Act under Project License PPL 70/7620 following an appropriate ethical review process at the Babraham Institute. No live vertebrates or higher invertebrates were used in this research.

\section{AUTHOR CONTRIBUTIONS}

$\mathrm{CH}$, JS, $\mathrm{AL}, \mathrm{DM}$, and $\mathrm{MC}$ conceived the study, designed the experiments, and wrote the manuscript. $\mathrm{CH}$ performed all experiments.

\section{REFERENCES}

1. Menon DK, Schwab K, Wright DW, Maas AI. Position statement: definition of traumatic brain injury. Arch Phys Med Rehabil. (2010) 91:1637-40. doi: 10.1016/j.apmr.2010.05.017

2. Maas AI, Menon DK, Steyerberg EW, Citerio G, Lecky F, Manley GT, et al. Collaborative european neuroTrauma effectiveness research in traumatic brain injury (CENTER-TBI): a Prospective longitudinal observational study. Neurosurgery. (2015) 76:67-80. doi: 10.1227/NEU.0000000000000575

3. Hill CS, Coleman MP, Menon DK. Traumatic axonal injury: mechanisms and translational opportunities. Trends Neurosci. (2016) 39:311-24. doi: 10.1016/j.tins.2016.03.002

4. Conforti L, Gilley J, Coleman MP. Wallerian degeneration: an emerging axon death pathway linking injury and disease. Nat Rev Neurosci. (2014) 15:394-409. doi: 10.1038/nrn3680

5. Wang J, Hamm RJ, Povlishock JT. Traumatic axonal injury in the optic nerve: evidence for axonal swelling, disconnection, dieback, and reorganization. $J$ Neurotrauma. (2011) 28:1185-98. doi: 10.1089/neu.2011.1756

6. Maxwell WL, Watt C, Graham DI, Gennarelli TA. Ultrastructural evidence of axonal shearing as a result of lateral acceleration of the head in non-human primates. Acta Neuropathol. (1993) 86:136-44. doi: 10.1007/BF00334880

7. Christman CW, Grady MS, Walker SA, Holloway KL, Povlishock JT. Ultrastructural studies of diffuse axonal injury in humans. J Neurotrauma. (1994) 11:173-86. doi: 10.1089/neu.1994.11.173

8. Smith DH, Wolf JA, Lusardi TA, Lee VM, Meaney DF. High tolerance and delayed elastic response of cultured axons to dynamic stretch injury. $J$ Neurosci. (1999) 19:4263-9. doi: 10.1523/JNEUROSCI.19-11-04263.1999

9. Büki A, Povlishock JT. All roads lead to disconnection? Traumatic axonal injury revisited. Acta Neurochir. (2006) 148:181-93. doi: 10.1007/s00701-005-0674-4

10. Smith DH, Hicks R, Povlishock JT. Therapy development for diffuse axonal injury. J Neurotrauma. (2013) 30:307-23. doi: 10.1089/neu.2012.2825

11. Zhai RG, Zhang F, Hiesinger PR, Cao Y, Haueter CM, Bellen HJ. NAD synthase nMNAT acts as a chaperone to protect against neurodegeneration. Nature. (2008) 452:887-91. doi: 10.1038/nature06721

12. Ali YO, McCormack R, Darr A, Zhai RG. Nicotinamide mononucleotide adenylyltransferase is a stress response protein regulated by the heat shock

\section{ACKNOWLEDGMENTS}

I would like to thank Vishnu Janardan for fly-keeping support, Elena Fineberg and Myriam Hemberger for histology support, Barbara Sobotic for flow-cytometry support, Alex Whitworth, Alvaro Sanchez-Martinez, and Victoria Hewitt for dopaminergic neuron staining support, Claire Durrant and other members of the Coleman lab for scientific discussions. I would also like to thank Marc Freeman and Frank Hirth for fly lines. $\mathrm{CH}$ is supported by a Wellcome Trust Ph.D. for Clinicians. DM is supported by a Senior Investigator Award from the National Institute for Health Research, UK (NIHR), by the Acute Brain Injury and Repair theme of the Cambridge NIHR Biomedical Research Centre, and a Framework Program 7 grant from the European Union (CENTER-TBI; Grant no: 602150). MC is funded by the John and Lucille van Geest Foundation.

\section{SUPPLEMENTARY MATERIAL}

The Supplementary Material for this article can be found online at: https://www.frontiersin.org/articles/10.3389/fneur. 2020.00401/full\#supplementary-material

factor/hypoxia-inducible factor $1 \alpha$ pathway. J Biol Chem. (2011) 286:1908999. doi: 10.1074/jbc.M111.219295

13. Di Stefano M, Nascimento-Ferreira I, Orsomando G, Mori V, Gilley J, Brown $\mathrm{R}$, et al. A rise in NAD precursor nicotinamide mononucleotide (NMN) after injury promotes axon degeneration. Cell Death Differ. (2015) 22:731-42. doi: $10.1038 /$ cdd.2014.164

14. Ali YO, Allen HM, Yu L, Li-Kroeger D, Bakhshizadehmahmoudi D, Hatcher A, et al. NMNAT2: hSP90 complex mediates proteostasis in proteinopathies. PLoS Biol. (2016) 14:e1002472. doi: 10.1371/journal.pbio.1 002472

15. Brazill JM, Li C, Zhu Y, Zhai RG. NMNAT: it's an nAD+ synthase... it's a chaperone... it's a neuroprotector. Curr Opin Genet Dev. (2017) 44:156-62. doi: 10.1016/j.gde.2017.03.014

16. Gilley J, Coleman MP. Endogenous nmnat2 is an essential survival factor for maintenance of healthy axons. PLoS Biol. (2010) 8:e1000300. doi: 10.1371/journal.pbio.1000300

17. Gilley J, Adalbert R, Yu G, Coleman MP. Rescue of peripheral and cNS axon defects in mice lacking nMNAT2. J Neurosci. (2013) 33:13410-3424. doi: 10.1523/JNEUROSCI.1534-13.2013

18. Milde S, Gilley J, Coleman MP. Axonal trafficking of nMNAT2 and its roles in axon growth and survival in vivo. Bioarchitecture. (2013) 3:133-40. doi: 10.4161/bioa.27049

19. Osterloh JM, Yang J, Rooney TM, Fox AN, Adalbert R, Powell EH, et al. dSarm/Sarm1 is required for activation of an injury-induced axon death pathway. Science. (2012) 337:481-4. doi: 10.1126/science.12 23899

20. Gilley J, Orsomando G, Nascimento-Ferreira I, Coleman MP. Absence of sARM1 rescues development and survival of nMNAT2-Deficient axons. Cell Rep. (2015) 10:1974-81. doi: 10.1016/j.celrep.2015.02.060

21. Loreto A, Di Stefano M, Gering M, Conforti L. Wallerian degeneration is executed by an nMN-SARM1-dependent late $\mathrm{ca}(2+)$ influx but only modestly influenced by mitochondria. Cell Rep. (2015) 13:2539-52. doi: 10.1016/j.celrep.2015.11.032

22. Neukomm LJ, Burdett TC, Seeds AM, Hampel S, Coutinho-Budd JC, Farley JE, et al. Axon death pathways converge on axundead to promote functional and structural axon disassembly. Neuron. (2017) 95:78-91.e5. doi: 10.1016/j.neuron.2017.06.031 
23. Loreto A, Hill CS, Hewitt VL, Orsomando G, Angeletti C, Gilley J, et al. Mitochondrial impairment activates the wallerian pathway through depletion of NMNAT2 leading to sARM1-dependent axon degeneration. Neurobiol Dis. (2020) 134:104678. doi: 10.1016/j.nbd.2019.104678

24. Xiong X, Hao Y, Sun K, Li J, Li X, Mishra B, et al. The highwire ubiquitin ligase promotes axonal degeneration by tuning levels of nmnat protein. PLoS Biol. (2012) 10:e1001440. doi: 10.1371/journal.pbio.1001440

25. Fang Y, Soares L, Teng X, Geary M, Bonini NM. A novel drosophila model of nerve injury reveals an essential role of nmnat in maintaining axonal integrity. Curr Biol. (2012) 22:590-5. doi: 10.1016/j.cub.2012.01.065

26. Ali YO, Li-Kroeger D, Bellen HJ, Zhai RG, Lu HC. NMNATs, evolutionarily conserved neuronal maintenance factors. Trends Neurosci. (2013) 36:632-40. doi: 10.1016/j.tins.2013.07.002

27. Zhai RG, Cao Y, Hiesinger PR, Zhou Y, Mehta SQ, Schulze KL, et al. Drosophila NMNAT maintains neural integrity independent of its NAD synthesis activity. PLoS Biol. (2006) 4:e416. doi: 10.1371/journal.pbio.0040416

28. Llobet Rosell A, Neukomm LJ. Axon death signalling in wallerian degeneration among species and in disease. Open Biol. (2019) 9:190118. doi: 10.1098/rsob.190118

29. Fox GB, Faden AI. Traumatic brain injury causes delayed motor and cognitive impairment in a mutant mouse strain known to exhibit delayed wallerian degeneration. J Neurosci Res. (1998) 53:718-727. doi: 10.1002/(SICI)1097-4547(19980915)53:6<718::AID-JNR9>3.0.CO;2-8

30. Henninger N, Bouley J, Sikoglu EM, An J, Moore CM, King JA, et al. Attenuated traumatic axonal injury and improved functional outcome after traumatic brain injury in mice lacking sarm1. Brain. (2016) 2016:1-12. doi: 10.1093/brain/aww001

31. Wu C, Waikar YP, Collins CS, DiAntonio A. Highwire function at the drosophila neuromuscular junction: spatial, structural, and temporal requirements. J Neurosci. (2005) 25:9557-66. doi: 10.1523/JNEUROSCI.2532-05.2005

32. Neukomm LJ, Burdett TC, Gonzalez MA, Züchner S, Freeman MR. Rapid in vivo forward genetic approach for identifying axon death genes in drosophila. Proc Natl Acad Sci USA. (2014) 111:9965-70. doi: 10.1073/pnas.1406230111

33. Neukomm LJ, Freeman MR. Diverse cellular and molecular modes of axon degeneration. Trends Cell Biol. (2014) 24:515-23. doi: 10.1016/j.tcb.2014.04.003

34. Babetto E, Beirowski B, Russler EV, Milbrandt J, DiAntonio A. The phr1 ubiquitin ligase promotes injury-induced axon self-destruction. Cell Rep. (2013) 3:1422-9. doi: 10.1016/j.celrep.2013.04.013

35. Grill B, Murphey RK, Borgen MA. The pHR proteins: intracellular signaling hubs in neuronal development and axon degeneration. Neural Dev. (2016) 11:8. doi: 10.1186/s13064-016-0063-0

36. Wan HI, DiAntonio A, Fetter RD, Bergstrom K, Strauss R, Goodman CS. Highwire regulates synaptic growth in drosophila. Neuron. (2000) 26:313329. doi: 10.1016/S0896-6273(00)81166-6

37. Collins CA, Wairkar YP, Johnson SL, DiAntonio A. Highwire restrains synaptic growth by attenuating a mAP kinase signal. Neuron. (2006) 51:57-69. doi: 10.1016/j.neuron.2006.05.026

38. Katzenberger RJ, Loewen CA, Wassarman DR, Petersen AJ, Ganetzky B, Wassarman DA. A drosophila model of closed head traumatic brain injury. Proc Natl Acad Sci. (2013) 110:E4152-E9. doi: 10.1073/pnas.1316895110

39. Barekat A, Gonzalez A, Mauntz RE, Kotzebue RW, Molina B, El-Mecharrafie $\mathrm{N}$, et al. Using drosophila as an integrated model to study mild repetitive traumatic brain injury. Sci Rep. (2016) 6:1-4. doi: 10.1038/srep25252

40. Fang Y, Bonini NM. Axon degeneration and regeneration: insights from drosophila models of nerve injury. Annu Rev Cell Dev Biol. (2012) 28:575-97. doi: 10.1146/annurev-cellbio-101011-155836

41. Gargano JW, Martin I, Bhandari P, MS G. Rapid iterative negative geotaxis (RING): a new method for assessing age-related locomotor decline in drosophila. Exp Gerontol. (2005) 45:125-30. doi: 10.1016/j.exger.2005.02.005
42. Nichols CD, Becnel J, Pandey. Methods to assay drosophila behavior. JoVE. (2012) 61:1-5. doi: 10.3791/3795

43. Mao Z, Davis RL. Eight different types of dopaminergic neurons innervate the drosophila mushroom body neuropil: anatomical and physiological heterogeneity. Front Neural Circuits. (2009) 3:5. doi: 10.3389/neuro.04.005.2009

44. Collins CA, DiAntonio A. Synaptic development: insights from drosophila Curr Opin Neurobiol. (2007) 17:35-42. doi: 10.1016/j.conb.2007.01.001

45. Katzenberger RJ, Chtarbanova S, Rimkus SA, Fischer JA, Kaur G, Seppala JM, et al. Death following traumatic brain injury in drosophila is associated with intestinal barrier dysfunction. Elife. (2015) 4:e04790. doi: 10.7554/eLife. 04790

46. Agrawal T, Hasan G. Maturation of a central brain flight circuit in drosophila requires fz2/Ca ${ }^{2}$ ? signaling. Elife. (2015) 4:e07046. doi: 10.7554/eLife.07046

47. Kretzschmar D, Hasan G, Sharma S, Heisenberger M, Benzer S. The swiss cheese mutant causes glial hyperwrapping and brain degeneration in drosophila. J Neurosci. (1997) 17:7425-32. doi: 10.1523/JNEUROSCI.17-19-07425.1997

48. Mutsuddi M, Nambu JR. Neural disease: drosophila degenerates for a good cause. Curr Biol. (1998) 8:R809-R11. doi: 10.1016/S0960-9822(07)00506-4

49. Lessing D, Bonini NM. Maintaining the brain: insight into human neurodegeneration from drosophila melanogaster mutants. Nat Rev Genet. (2009) 2009:359-70. doi: 10.1038/nrg2563

50. Impellizzeri D, Campolo M, Bruschetta G, Crupi R, Cordaro M, Paterniti I, et al. Traumatic brain injury leads to development of parkinson's disease related pathology in mice. Front Neurosci. (2016) 10:458. doi: 10.3389/fnins.2016.00458

51. Whitworth AJ, Theodore DA, Greene JC, Beneš H, Wes PD, Pallanck LJ. Increased glutathione s-transferase activity rescues dopaminergic neuron loss in a drosophila model of parkinson's disease. Proc Natl Acad Sci. (2005) 102:8024-9. doi: 10.1073/pnas.0501078102

52. Hutson CB, Lazo CR, Mortazavi F, Giza CC, Hovda D, Chesselet MF. Traumatic brain injury in adult rats causes progressive nigrostriatal dopaminergic cell loss and enhanced vulnerability to the pesticide paraquat. J Neurotrauma. (2011) 28:1783-801. doi: 10.1089/neu.2010.1723

53. Hirth F. Drosophila melanogaster in the study of human neurodegeneration. CNS \& Neurological Disorders-Drug Targets. (2010) 9:504-23. doi: 10.2174/187152710791556104

54. Lavado-Roldán A, Fernández-Chacón R. Two for the price of one: a Neuroprotective chaperone kit within nAD synthase protein nMNAT2. PLoS Biol. (2016) 14:e1002522. doi: 10.1371/journal.pbio.1002522

55. Harwell CS, Coleman MP. Synaptophysin depletion and intraneuronal a $\beta$ in organotypic hippocampal slice cultures from huAPP transgenic mice. $\mathrm{Mol}$ Neurodeg. (2016) 11:44. doi: 10.1186/s13024-016-0110-7

56. Bae JR, Kim SH. Synapses in neurodegenerative diseases. BMB Rep. (2017) 50:237. doi: 10.5483/BMBRep.2017.50.5.038

57. Zang S, Ali YO, Ruan K, Zhai RG. Nicotinamide mononucleotide adenylyltransferase maintains active zone structure by stabilizing bruchpilot. EMBO Rep. (2013) 14:87-94. doi: 10.1038/embor.20 12.181

Conflict of Interest: The authors declare that the research was conducted in the absence of any commercial or financial relationships that could be construed as a potential conflict of interest.

Copyright (c) 2020 Hill, Sreedharan, Loreto, Menon and Coleman. This is an openaccess article distributed under the terms of the Creative Commons Attribution License (CC BY). The use, distribution or reproduction in other forums is permitted, provided the original author(s) and the copyright owner(s) are credited and that the original publication in this journal is cited, in accordance with accepted academic practice. No use, distribution or reproduction is permitted which does not comply with these terms. 